\title{
Differences between clinic blood pressure and morning home blood pressure, as shown by Bland-Altman plots, in a large observational study (HONEST study)
}

\author{
Kazuyuki Shimada ${ }^{1}, K_{\text {Kazuomi Kario }}^{2}$, Toshio Kushiro ${ }^{3}$, Satoshi Teramukai ${ }^{4}$, Yusuke Ishikawa ${ }^{5}$, \\ Fumiaki Kobayashi ${ }^{6}$ and Ikuo Saito ${ }^{7}$
}

When interpreting home blood pressure (BP) measurements in hypertensive patients, differences between clinic and home BP should be noted. To investigate the differences between clinic and morning home BP in hypertensive patients, we analyzed clinic systolic BP (CSBP) and morning home systolic BP (MHSBP) data from the large-scale observational HONEST (Home BP measurement with Olmesartan Naive patients to Establish Standard Target blood pressure) study ( $n=21340$ ), using BP measurements obtained before starting olmesartan administration. We generated Bland-Altman plots, with the horizontal axis representing mean CSBP and MHSBP ([CSBP+MHSBP]/2) and the vertical axis representing the difference between CSBP and MHSBP (CSBP-MHSBP). We also did simulation experiments to explore factors affecting the results of the Bland-Altman plots. The difference between CSBP and MHSBP increased as the mean of the two values increased, and when the mean of CSBP and MHSBP was close to $140 \mathrm{~mm} \mathrm{Hg}$, the difference was theoretically 0 in average, although large interindividual BP variability existed in this BP range. Results were unaffected by factors such as previous antihypertensive treatment, age and concomitant diabetes or chronic kidney disease. Bland-Altman plots generated from simulated data of normal distribution showed that the slope of the regression line sloped upward, consistent with the results of the HONEST study, when the interindividual BP variability of MHSBP was less than that of CSBP. In conclusion, differences between mean CSBP and MHSBP may be caused by large interindividual variability in CSBP. Therefore, the differences between MHSBP and CSBP may vary between patient groups, which should be noted in the management of hypertension. Hypertension Research (2015) 38, 876-882; doi:10.1038/hr.2015.88; published online 6 August 2015

Keywords: ambulatory; blood pressure; blood pressure monitoring; variation

\section{INTRODUCTION}

Home blood pressure (BP) measurements are superior to clinic $\mathrm{BP}$ measurements in terms of reproducibility ${ }^{1-3}$ and prediction of prognosis. ${ }^{4-6}$ Moreover, because home BP can be easily measured any number of times, it is suitable for long-term observation. Therefore, home BP is useful as an indicator of BP control in clinical practice.

In Japan, the measurement of home BP is relatively well established, and it is used in combination with clinic BP measurements to assess BP control. However, many clinical studies that investigated the effects of antihypertensive treatment on the prevention of cardiovascular events have used only clinic BP as an indicator of the efficacy of treatment, ${ }^{7-12}$ because the use of clinic BP is the gold standard in the evaluation of BP control.

It is well known that there is discrepancy between home and clinic $\mathrm{BP}$ values in the same patient, and, in most cases, clinic BP is higher than home BP because of the white coat effect. ${ }^{13} \mathrm{~A}$ meta-analysis of 13 studies showed that clinic BP is higher than home BP by an average of $5.3 \mathrm{~mm} \mathrm{Hg}$ for systolic BP and $3.1 \mathrm{~mm} \mathrm{Hg}$ for diastolic BP. ${ }^{14}$ Moreover, the cutoff values for home BP as diagnostic criteria to define hypertension are lower than those of clinic BP, although they are slightly inconsistent depending on the interpretation of evidence (i.e. $125 / 80 \mathrm{~mm} \mathrm{Hg}$ as defined by the World Health Organization ${ }^{15}$ and $135 / 85 \mathrm{~mm} \mathrm{Hg}$ as defined by the guidelines of Europe and Japan ${ }^{16,17}$ ).

As well as the definition of diagnostic criteria for hypertension, physicians are also concerned about the determination of target home $\mathrm{BP}$ values. Although the diagnostic criteria might be closely related to the target home BP values, BP values should be considered independently. However, the evidence is even scarcer with regard to target home BP.

The differences between home BP and clinic BP has already been reported using the results of a study that used data from the general

${ }^{1}$ Shin-Oyama City Hospital, Oyama, Japan; ${ }^{2}$ Division of Cardiovascular Medicine, Department of Medicine, Jichi Medical University School of Medicine, Shimotsuke, Japan, ${ }^{3}$ The Life Planning Center Foundation, Tokyo, Japan; ${ }^{4}$ Department of Biostatistics, Graduate School of Medical Science, Kyoto Prefectural University of Medicine, Kyoto, Japan; ${ }^{5}$ Daiichi Sankyo, Tokyo, Japan; ${ }^{6}$ Daiichi Sankyo Development, Gerrards Cross, UK and ${ }^{7}$ Keio University, Yokohama, Japan

Correspondence: Dr K Shimada, Shin-Oyama City Hospital, 1-1-5 Wakagicho, Oyama, Tochigi 323-0028, Japan.

E-mail: kazuyuki@hospital.oyama.tochigi.jp

Part of the work was presented at the 36th Annual Scientific Meeting of the Japanese Society of Hypertension.

Received 24 December 2014; revised 1 May 2015; accepted 12 May 2015; published online 6 August 2015 
population and a systematic review of several studies that used data from the general population and/or hypertensive patients. ${ }^{6,14}$ However, few studies have compared them, and current evidence remains limited. Moreover, no reports have examined the differences in detail using data from a large-scale study involving hypertensive patients.

The Home BP measurement with Olmesartan Naive patients to Establish Standard Target blood pressure (HONEST) study was undertaken to investigate the relationship between on-treatment home $\mathrm{BP}$ and the occurrence of cardiovascular, renal and other events in patients with essential hypertension who started antihypertensive treatment with olmesartan, including those whose current antihypertensive treatment was supplemented or replaced by olmesartan therapy, ${ }^{18}$ and the main results of the HONEST study have been reported. ${ }^{19}$ In the present study, we used data from $>20000$ patients to evaluate the discrepancy and the relationship between morning home BP and clinic BP in hypertensive patients, neither of which have been fully examined in large-scale studies. We used baseline BP, because it reflects individual differences and is useful to evaluate the effects of previous and concomitant antihypertensive treatment. Although evening home BP was also collected, in this analysis we used only morning home $\mathrm{BP}$, because at enrollment evening home $\mathrm{BP}$ was not obligatory.

\section{METHODS}

\section{Study design}

The aims and protocol of the HONEST study have already been reported. ${ }^{18}$ HONEST is a large-scale prospective observational study involving patients receiving olmesartan-based treatment with a 2-year follow-up and was registered at http://www.umin.ac.jp/ctr/index.htm with the unique trial number UMIN000002567. The study protocol was approved by the Ethical Committee of Daiichi Sankyo and by the review boards of the institutions at their discretion; it conforms with the Pharmaceutical Affairs Laws of Japan and was approved by the Ministry of Health, Labour and Welfare of Japan before commencement. The study was carried out in registered medical institutions, in compliance with Good Post-marketing Study Practice in Japan and internal regulations for clinical studies at each institution. Data were collected through the Internet using a central electronic data-capturing system (PostMaNet; Fujitsu FIP, Tokyo, Japan) that had been validated.

\section{Patients}

The HONEST study included patients with essential hypertension who had never been treated with olmesartan (as reported by physicians, with no specific $\mathrm{BP}$ range). Patients were excluded if they had a history of recent cardiovascular events or a planned cardiovascular intervention. With the exception of prior use of olmesartan, no restriction was placed on prior or concomitant antihypertensive drugs during the study.

\section{BP measurement}

In the present analysis, we used baseline BP to evaluate the effects of previous and concomitant antihypertensive treatment on the differences between the home $\mathrm{BP}$ and clinic $\mathrm{BP}$. Home $\mathrm{BP}$ was measured by patients using a home $\mathrm{BP}$ measuring device with an upper arm cuff. Home BP measuring devices available in Japan are validated and approved by the Ministry of Health, Labour and Welfare of Japan, and they comply with the Association for the Advancement of Medical Instrumentation ${ }^{20}$ or European standards. ${ }^{21}$ Patients recorded their morning home $\mathrm{BP}$ on $\geqslant 2$ days in the 28 -day period before starting olmesartan therapy in real-world setting. At each time point, patients measured their BP two times. In the present analysis, we used the mean of two daily morning home BP values (the first measurements). When patients had recorded their $\mathrm{BP}$ on more than 2 different days, the two measurements for the days closest to the start of olmesartan therapy were used. Clinic BP was measured according to the usual methods of each institution under daily clinical practice, so as to obtain data in real-world clinical practice. We used one clinic systolic BP (CSBP) measurement obtained within the 28 days before the start of olmesartan therapy.

\section{Data analysis}

To investigate the agreement between CSBP and morning home systolic BP (MHSBP), we performed a Bland-Altman (BA) analysis; this method is used to compare two different measurement techniques. ${ }^{22,23}$ Accordingly, we generated BA plots, with the horizontal axis representing the mean of CSBP and MHSBP $([\mathrm{CSBP}+\mathrm{MHSBP}] / 2)$ and the vertical axis representing the difference between CSBP and MHSBP (CSBP-MHSBP). Subsequently, to verify the differences between each BP (CSBP or MHSBP) and the difference between them (CSBP-MHSBP), we performed additional analyses, with the horizontal axis representing either CSBP or MHSBP alone.

In the BA plots, we plotted the moving averages of the difference between CSBP and MHSBP as the values for the horizontal axis (mean of CSBP and MHSBP) by $5 \mathrm{~mm} \mathrm{Hg}$. For instance, for BP $110 \mathrm{~mm} \mathrm{Hg}$, the average of the values in a range between 105 and $115 \mathrm{~mm} \mathrm{Hg}$ was plotted; next, for $\mathrm{BP} 115 \mathrm{~mm} \mathrm{Hg}$, the average of values between 110 and $120 \mathrm{~mm} \mathrm{Hg}$ was plotted; and so forth.

We also conducted univariate regression analysis of the mean of CSBP and MHSBP on the difference between CSBP and MHSBP. Furthermore, we conducted multivariate regression analysis to explore the effects of various factors (previous antihypertensive treatment, age and concomitant diabetes or chronic kidney disease (CKD)) on the slope of the regression line between difference and the mean of CSBP and MHSBP.

The results of the BA analysis showed a positive correlation between the mean of CSBP and MHSBP ([CSBP+MHSBP $] / 2)$ and the difference between CSBP and MHSBP (CSBP-MHSBP). We hypothesized that this may have been caused by differences in interindividual BP variability between CSBP and MHSBP, thus we conducted simulation experiments using the standard deviation (s.d.) of each BP. Four scenarios, that is, (s.d. of CSBP, s.d. of MHSBP $)=(19.0,16.4),(19.0,19.0),(16.4,16.4)$, and $(16.4,19.0)$, were used to generate simulation data at random from normal distribution with a single mean value $(150 \mathrm{~mm} \mathrm{Hg})$.

CSBP and MHSBP with s.d. in patients with and without a history of cerebro- or cardiovascular disease, concomitant diabetes and CKD were also evaluated.

Data are reported as mean \pm s.d. All tests were two-sided and carried out to the 5\% level of significance. SAS release 9.2 software (SAS Institute, Cary, NC, USA) was used for all statistical analyses.

\section{RESULTS}

\section{Baseline characteristics}

The baseline characteristics of the patients are presented in Table 1. From 3039 medical institutions throughout Japan, 22373 patients were enrolled in the study. The present analysis included data from 21340 patients, after excluding patients for reasons such as withdrawal of consent. Patients were divided into those who had been untreated $(n=10609)$ and those who had been treated $(n=10731)$ at baseline. Their mean age was $62.8 \pm 12.0$ and $66.8 \pm 11.4$ years, respectively. CSBP and MHSBP were $159.2 \pm 18.0$ and $156.4 \pm 15.5 \mathrm{~mm} \mathrm{Hg}$ in untreated patients and $148.1 \pm 18.4$ and $146.9 \pm 16.0 \mathrm{~mm} \mathrm{Hg}$ in treated patients, respectively.

\section{Agreement between CSBP and MHSBP}

Figures $1 \mathrm{a}$ and $\mathrm{b}$ show the BA plots for treated and untreated patients. The percentage of patients whose BP was $-5 \leqslant($ CSBP-MHSBP) $\leqslant 5 \mathrm{~mm} \mathrm{Hg}$ and $-10 \leqslant($ CSBP-MHSBP $) \leqslant 10 \mathrm{~mm} \mathrm{Hg}$ was $36.7 \%$ and $59.5 \%$ in untreated patients and $32.5 \%$ and $54.5 \%$ in treated patients, respectively (Table 1). The slope of the regression line between the difference and the mean of CSBP and MHSBP showed an upward trend. The difference between CSBP and MHSBP was positive (CSBP $>$ MHSBP) when the mean of CSBP and MHSBP was high, whereas it was negative $(\mathrm{CSBP}<\mathrm{MHSBP})$ when the mean of CSBP and MHSBP was low. 
Table 1 Baseline characteristics

\begin{tabular}{|c|c|c|c|c|}
\hline No. of subjects (\%) or mean \pm s.d. & All $(\mathrm{n}=21340)$ & Untreated $(n=10609)$ & Treated $(\mathrm{n}=10731)$ & P-value (untreated vs. treated) \\
\hline Women & $10784(50.5)$ & $5389(50.8)$ & $5395(50.3)$ & 0.4461 \\
\hline Age (years) & $64.8 \pm 11.9$ & $62.8 \pm 12.0$ & $66.8 \pm 11.4$ & $<0.0001$ \\
\hline Age range (years) & $16-100$ & $16-97$ & $25-100$ & \\
\hline Body mass index $\left(\mathrm{kg} \mathrm{m}^{-2}\right)$ & $24.31 \pm 3.70$ & $24.17 \pm 3.66$ & $24.44 \pm 3.74$ & $<0.0001$ \\
\hline Alcohol drinkers & $3439(16.1)$ & $1742(16.4)$ & $1697(15.8)$ & $<0.0001$ \\
\hline Current smokers & 2618 (12.3) & $1487(14.0)$ & $1131(10.5)$ & $<0.0001$ \\
\hline \multicolumn{5}{|l|}{ History } \\
\hline Cerebro- or cardiovascular disease & $2241(10.5)$ & $583(5.5)$ & $1658(15.5)$ & $<0.0001$ \\
\hline Cerebrovascular disease & $1415(6.6)$ & 411 (3.9) & $1004(9.4)$ & $<0.0001$ \\
\hline Cardiovascular disease & $966(4.5)$ & 201 (1.9) & $765(7.1)$ & $<0.0001$ \\
\hline \multicolumn{5}{|l|}{ Complications } \\
\hline Dyslipidemia & $9484(44.4)$ & $3937(37.1)$ & $5547(51.7)$ & $<0.0001$ \\
\hline Diabetes mellitus & $4364(20.4)$ & $1592(15.0)$ & $2772(25.8)$ & $<0.0001$ \\
\hline Chronic kidney disease & $4283(20.1)$ & $1573(14.8)$ & $2710(25.3)$ & $<0.0001$ \\
\hline \multicolumn{5}{|l|}{ Antihypertensive drugs used } \\
\hline Calcium channel blocker & $7689(36.0)$ & $0(0.0)$ & 7689 (71.7) & $<0.0001$ \\
\hline Angiotensin receptor blocker (except olmesartan) & $4535(21.3)$ & $0(0.0)$ & $4535(42.3)$ & $<0.0001$ \\
\hline$\beta$-Blocker & $1336(6.3)$ & $0(0.0)$ & $1336(12.4)$ & $<0.0001$ \\
\hline Diuretic & $1230(5.8)$ & $0(0.0)$ & $1230(11.5)$ & $<0.0001$ \\
\hline Angiotensin-converting enzyme inhibitor & $780(3.7)$ & $0(0.0)$ & $780(7.3)$ & $<0.0001$ \\
\hline$\alpha$-Blocker & $454(2.1)$ & $0(0.0)$ & $454(4.2)$ & $<0.0001$ \\
\hline Others & $90(0.4)$ & $0(0.0)$ & $90(0.8)$ & $<0.0001$ \\
\hline \multicolumn{5}{|l|}{ Clinic measurements } \\
\hline Systolic blood pressure $(\mathrm{mm} \mathrm{Hg})$ & $153.6 \pm 19.0$ & $159.2 \pm 18.0$ & $148.1 \pm 18.4$ & $<0.0001$ \\
\hline Diastolic blood pressure $(\mathrm{mm} \mathrm{Hg})$ & $87.1 \pm 13.4$ & $91.4 \pm 12.6$ & $82.9 \pm 12.7$ & $<0.0001$ \\
\hline Pulse rate (beats per min) & $74.1 \pm 11.2$ & $74.6 \pm 11.0$ & $73.5 \pm 11.4$ & $<0.0001$ \\
\hline \multicolumn{5}{|l|}{ Home measurements $\mathrm{a}^{\mathrm{a}}$} \\
\hline Systolic blood pressure $(\mathrm{mm} \mathrm{Hg})$ & $151.6 \pm 16.4$ & $156.4 \pm 15.5$ & $146.9 \pm 16.0$ & $<0.0001$ \\
\hline Diastolic blood pressure $(\mathrm{mm} \mathrm{Hg})$ & $87.1 \pm 11.8$ & $90.7 \pm 11.2$ & $83.5 \pm 11.3$ & $<0.0001$ \\
\hline Pulse rate (beats per min) & $70.8 \pm 10.0$ & $71.9 \pm 9.8$ & $69.7 \pm 10.0$ & $<0.0001$ \\
\hline Patients with blood pressure $-5 \leqslant($ CSBP - MHSBP $) \leqslant 5 \mathrm{~mm} \mathrm{Hg}$ & $7384(34.6)$ & $3893(36.7)$ & 3491 (32.5) & $<0.0001$ \\
\hline Patients with blood pressure $-10 \leqslant(\mathrm{CSBP}-\mathrm{MHSBP}) \leqslant 10 \mathrm{~mm} \mathrm{Hg}$ & $12165(57.0)$ & $6317(59.5)$ & $5848(54.5)$ & $<0.0001$ \\
\hline
\end{tabular}

Abbreviations: CSBP, clinic systolic blood pressure; MHSBP, morning home blood pressure

a Mean of two daily morning BP measurements.

In the subsequent analyses with the horizontal axis representing either CSBP (Figures 1c and d) or MHSBP (Figures 1e and $\mathrm{f}$ ) alone, a similar trend was confirmed; the difference between CSBP and MHSBP was greater when CSBP was high or when MHSBP was low.

In this study, home BP was measured according to each patient's own method. To clarify the effects of this on the results, we used data from approximately $80 \%$ of patients who measured home BP according to the methods defined by the Japanese Society Hypertension, ${ }^{17}$ and generated BA plots with a regression line (data not shown). The slope of the regression line was almost the same as that of all patients.

Effects of various factors on the slope of the regression line between difference and mean of CSBP and MHSBP

Table 2 shows the results of multiple linear regression analyses using the factors previous antihypertensive treatment, age and concomitant diabetes or CKD, which can affect the differences between CSBP and MHSBP. No significant difference in the slope of the regression line was observed with any of the factors, indicating that no factor affected the differences between CSBP and MHSBP.
Differences between CSBP and MHSBP stratified according to the mean of CSBP and MHSBP

Table 3 shows the differences between CSBP and MHSBP (CSBP-MHSBP) stratified according to the mean of CSBP and MHSBP $([\mathrm{CSBP}+\mathrm{MHSBP}] / 2)$ in three $5-\mathrm{mm} \mathrm{Hg}$ ranges $(>130-135 \mathrm{~mm} \mathrm{Hg}, \quad>135-140 \mathrm{~mm} \mathrm{Hg}$, and $>140-145 \mathrm{~mm} \mathrm{Hg})$. The differences between CSBP and MHSBP were near 0 , indicating that the two values are almost equal, when the mean systolic BP was $135-145 \mathrm{~mm} \mathrm{Hg}$ in treated patients and $130-140 \mathrm{~mm} \mathrm{Hg}$ in treated patients with diabetes or CKD.

Differences in the interindividual BP variability of CSBP and MHSBP and their effects on the slope of the regression line In the present analysis using data from 21340 patients, the s.d. of CSBP and MHSBP were 19.0 and $16.4 \mathrm{~mm} \mathrm{Hg}$, respectively. Although the MHSBP value used in this analysis was the mean MHSBP over 2 days, the s.d. of MHSBP on the first day only $(17.3 \mathrm{~mm} \mathrm{Hg})$ or the second day only $(17.6 \mathrm{~mm} \mathrm{Hg})$ was also less than that of CSBP. 

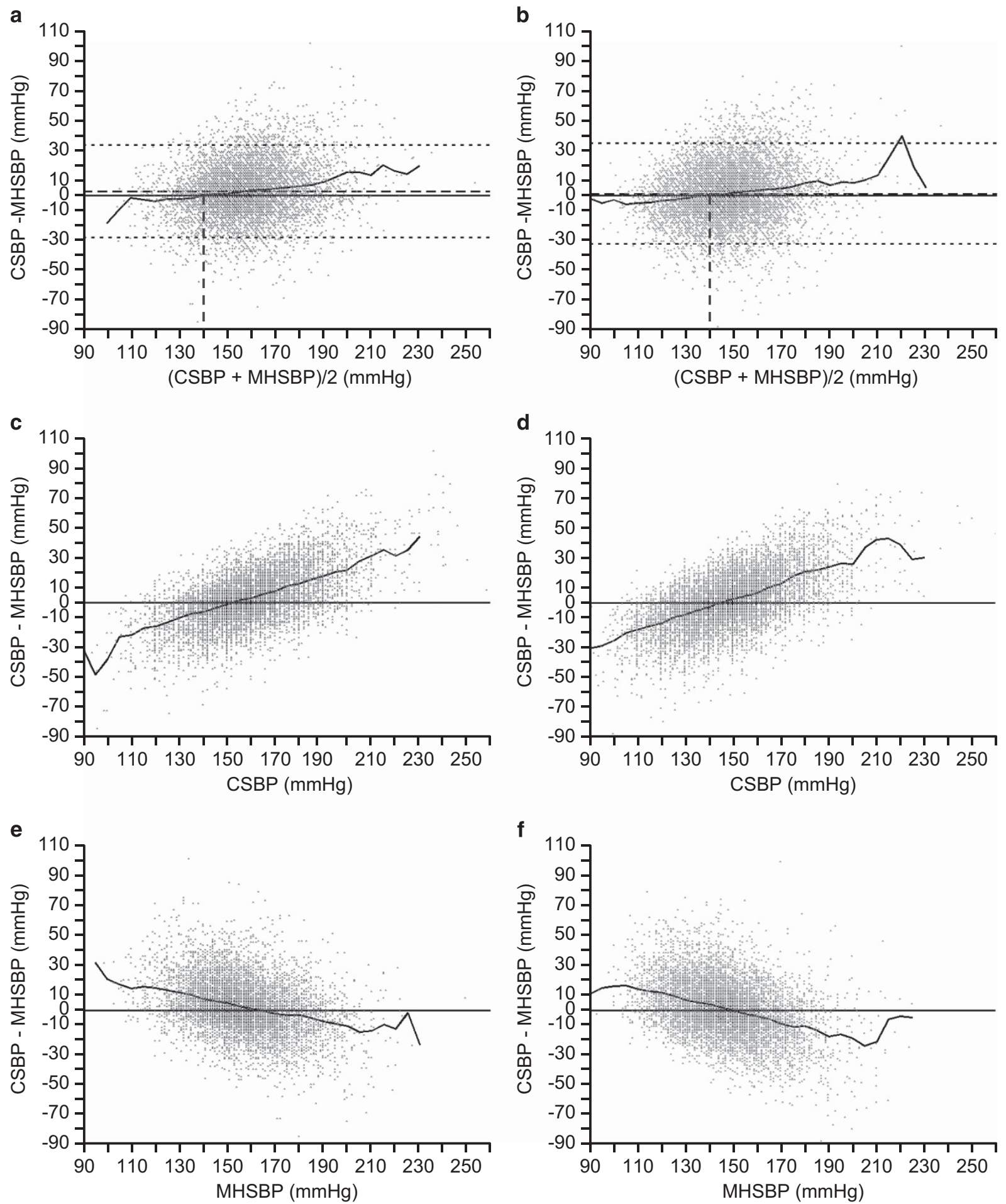

Figure 1 Bland-Altman (BA) plots showing the differences between the mean of clinic systolic blood pressure (CSBP) and morning home systolic blood pressure (MHSBP) and the difference between CSBP and MHSBP at baseline. (a) untreated patients $(n=10609)$ and (b) treated patients $(n=10731)$. $P=0.826$ for the interaction between (CSBP+MHSBP)/2 for untreated patients and (CSBP+MHSBP)/2 for treated patients. Calculated moving averages of the difference between CSBP and MHSBP as the values in the horizontal axis (mean of CSBP and MHSBP) increased by $5 \mathrm{~mm} \mathrm{Hg}$, and plotted these continuously in the BA plots. Results of subsequent analyses, with the horizontal axis representing CSBP (c, untreated patients; $\mathbf{d}$, treated patients) and MHSBP (e, untreated patients; $\mathbf{f}$, treated patients).

Figure 2 shows BA plots generated from the simulation experiments, with estimated regression lines. As shown in Figure 2a, when the s.d. of MHSBP is less than that of CSBP, the regression line has an upward slope, which is consistent with the results of the present analysis. On the other hand, when the s.d. are equal, the slope of the line is 0 (Figures $2 b$ and $c$ ), and when the s.d. of CSBP is less than that of MHSBP, the line has a downward slope (Figure 2d). 
Effects of previous or concomitant diseases on s.d. of CSBP and MHSBP

Table 4 shows mean CSBP and MHSBP with s.d. in patients with and without a history of cerebro- or cardiovascular disease, concomitant diabetes and CKD. In all subgroups, mean CSBP and MHSBP were similar, and there was no substantial difference in s.d.

\section{DISCUSSION}

We consider that this report provides abundant information on the differences between CSBP and MHSBP. The results of the BA analysis showed a tendency for the difference between CSBP and MHSBP to increase when the mean of CSBP and MHSBP was high. Moreover, when the mean of CSBP and MHSBP was close to $140 \mathrm{~mm} \mathrm{Hg}$, the difference was theoretically 0 in average, although there was large interindividual BP variability. The slope of the regression line was unaffected by factors such as previous antihypertensive treatment, age and concomitant diabetes or CKD.

In the present analysis, we hypothesized that there is one BP (true value) that can predict cardiovascular events, and clinic BP and morning home BP measurements are methods to identify the true value. Therefore, we used BA plots, which are visual representations of the agreement between two different measurement techniques. If both clinic BP and morning home BP measurements were the true value, there should be no difference between the two measurements. However, the results of the analysis showed that the two measurements do not always agree, and the regression line indicates a positive correlation. We assumed that this may be the result of interindividual BP variability between CSBP and MHSBP, thus we conducted simulation analyses. We also confirmed that the differences were unaffected by factors such as a history of cerebro- or cardiovascular disease and concomitant diseases.

Table 2 Effects of various factors on the slope of the regression line between the difference and the mean of clinic systolic blood pressure and morning home systolic blood pressure

\begin{tabular}{lccc}
\hline Factor & $\mathrm{n}$ & Slope of regression line $(95 \% \mathrm{Cl})$ & P-value \\
\hline Untreated & 10609 & $0.19(0.17-0.21)$ & 0.8260 \\
Treated & 10731 & $0.18(0.16-0.20)$ & \\
Age $<65$ years & 9817 & $0.17(0.15-0.18)$ & 0.0688 \\
Age $\geqslant 65$ years & 11523 & $0.19(0.17-0.21)$ & \\
No diabetes & 16976 & $0.19(0.17-0.20)$ & 0.0633 \\
Diabetes & 4364 & $0.16(0.12-0.20)$ & \\
No CKD & 16901 & $0.18(0.17-0.20)$ & 0.4998 \\
CKD & 4238 & $0.17(0.14-0.20)$ & \\
\hline
\end{tabular}

Abbreviations: $\mathrm{Cl}$, confidence interval; CKD, chronic kidney disease.
In a meta-analysis of several studies, BA analysis of the differences between home systolic BP and CSBP has shown a significant correlation between (clinic $\mathrm{BP}+$ home $\mathrm{BP}) / 2$ and clinic $\mathrm{BP}$-home $\mathrm{BP}$ in untreated hypertensive patients, and that the difference in $\mathrm{BP}$ increases as mean BP increases. ${ }^{14}$ In the Finn-HOME study, which involved 2051 individuals from the general adult population, BA analysis was also performed for clinic BP and home BP: a slight but significant positive correlation was found between (clinic BP+home $\mathrm{BP}) / 2$ and clinic BP-home BP, for both systolic and diastolic BP, and the difference in BP became greater as mean BP increased. ${ }^{24}$ Although these studies aimed to clarify the discrepancy between clinic and home $\mathrm{BP}$, the results are almost consistent with those of the present study, in respect that CSBP is higher than MHSBP when mean BP is high, whereas MHSBP is higher than CSBP when mean BP is low. In addition, the previous studies of the differences between clinic BP and home BP were mostly carried out in the general population. ${ }^{5,24}$ These studies provide valuable information; however, clinicians want to know the level of discrepancy between clinic and home BP in patients with hypertension who need treatment too. In this respect, the present study provides unique and sound evidence that differs from the findings of previous studies involving subjects from the general population.

In the J-HOME study, which involved 3308 patients with hypertension, BA plots were generated with the horizontal axis representing either CSBP or MHSBP alone, instead of their average. The observed tendency of the discrepancy between CSBP and MHSBP was similar to the results of the present study. In addition, the results of the J-HOME study showed that the difference between CSBP and MHSBP is increased in older patients. ${ }^{25}$

It should be noted that the above simulation is based on the assumption that average values of the CSBP and MHSBP are similar. In the present study, there was only a slight difference between CSBP and MHSBP values. However, several previous studies have reported that clinic BP is higher than home BP, whereas some studies in Japan showed that there was only a small difference (Supplementary Table). Similarly, the finding that s.d. of clinic BP is greater than that of home BP is consistent with many studies in Japan, whereas the results were opposite in Western studies (Supplementary Table). This may be because of the difference in regions, types of devices used and methods of BP measurement, which reflect real-world clinical practice. Further studies are necessary to clarify this issue.

The present study has several limitations. First, we used baseline BP that was measured before start of the study treatment. Therefore, home BP measured by patients may not fully comply with the study protocol. The reason we used baseline BP is because it reflects individual differences and is useful to evaluate the effects of previous and concomitant antihypertensive treatment, as compared with BP during the follow-up period. After the study completion, we confirmed that about $80 \%$ of patients complied with the BP measuring

Table 3 Differences between CSBP and MHSBP stratified according to mean of CSBP and MHSBP in three ranges ( $>130-135,>135-140$, $>140-145 \mathrm{~mm} \mathrm{Hg}$ )

\begin{tabular}{|c|c|c|c|c|c|c|c|c|c|c|}
\hline \multirow[b]{2}{*}{ Mean of CSBP and MHSBP } & \multicolumn{2}{|c|}{ All } & \multicolumn{2}{|c|}{ Untreated } & \multicolumn{2}{|c|}{ Treated } & \multicolumn{2}{|c|}{ Treated and DM } & \multicolumn{2}{|c|}{ Treated and CKD } \\
\hline & $n$ & Difference ${ }^{a}$ & $n$ & Difference ${ }^{\mathrm{a}}$ & $n$ & Difference $^{a}$ & $\mathrm{n}$ & Difference ${ }^{a}$ & $\mathrm{n}$ & Difference $^{\mathrm{a}}$ \\
\hline$>130-135$ & 1077 & -1.6 & 249 & -2.4 & 828 & -1.4 & 237 & -2.7 & 225 & -0.5 \\
\hline$>135-140$ & 1621 & -0.6 & 453 & -1.9 & 1168 & -0.1 & 304 & 0.5 & 285 & 0.6 \\
\hline$>140-145$ & 2258 & 0.4 & 806 & 1.2 & 1452 & 0.0 & 413 & 0.4 & 336 & 1.4 \\
\hline
\end{tabular}

Abbreviations: CKD, chronic kidney disease; CSBP, clinic systolic blood pressure; DM, diabetes mellitus; MHSBP, morning home systolic blood pressure.

a Mean of the difference between CSBP and MHSBP. 
a

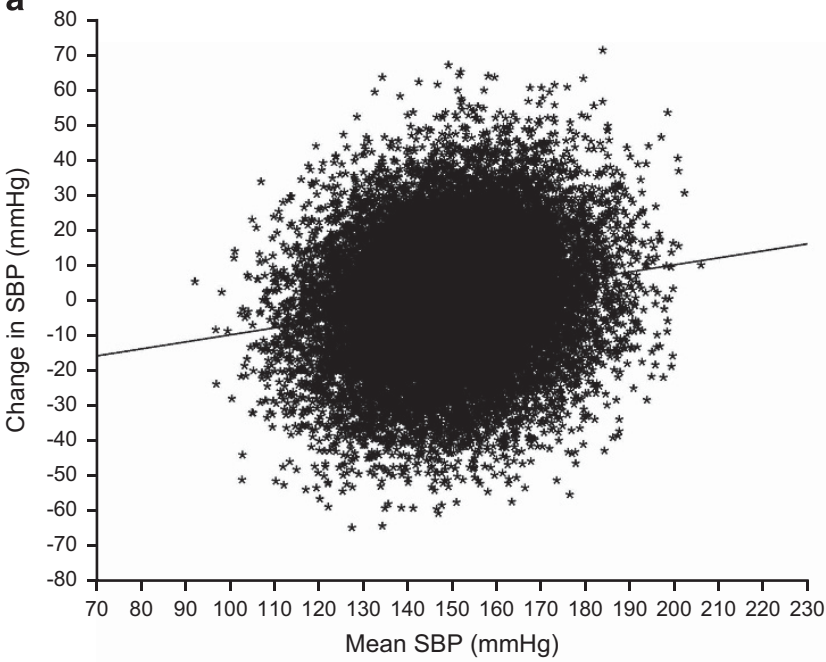

C

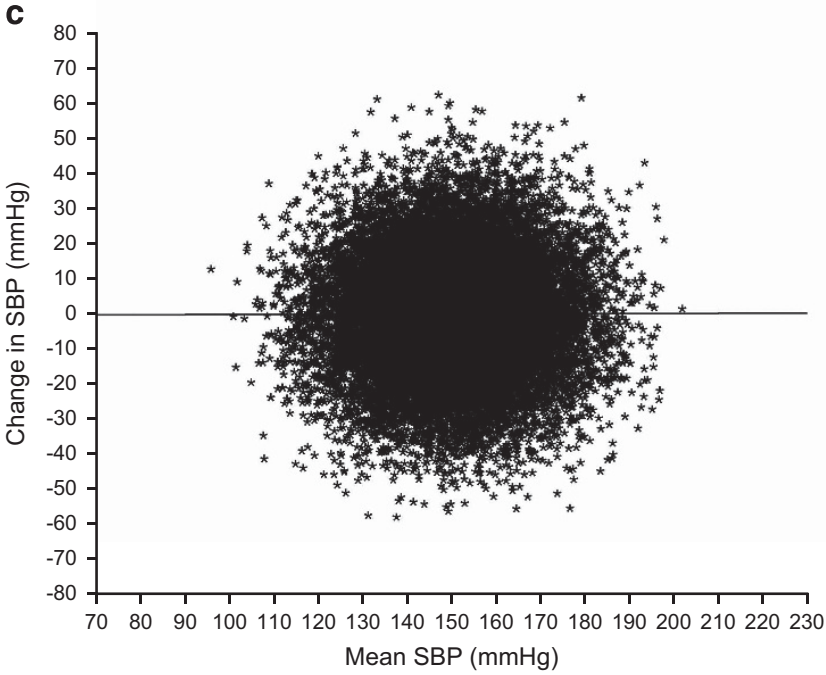

b

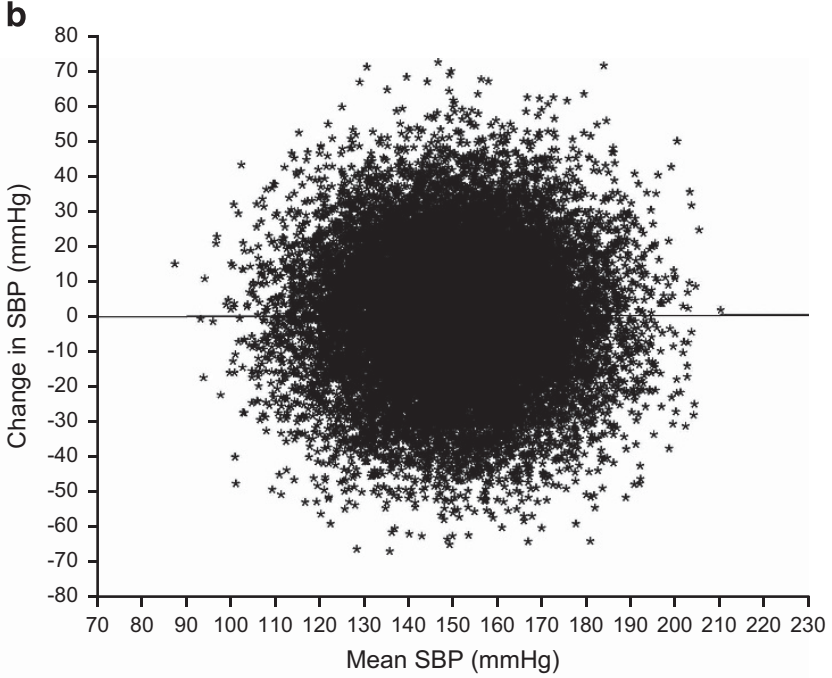

d

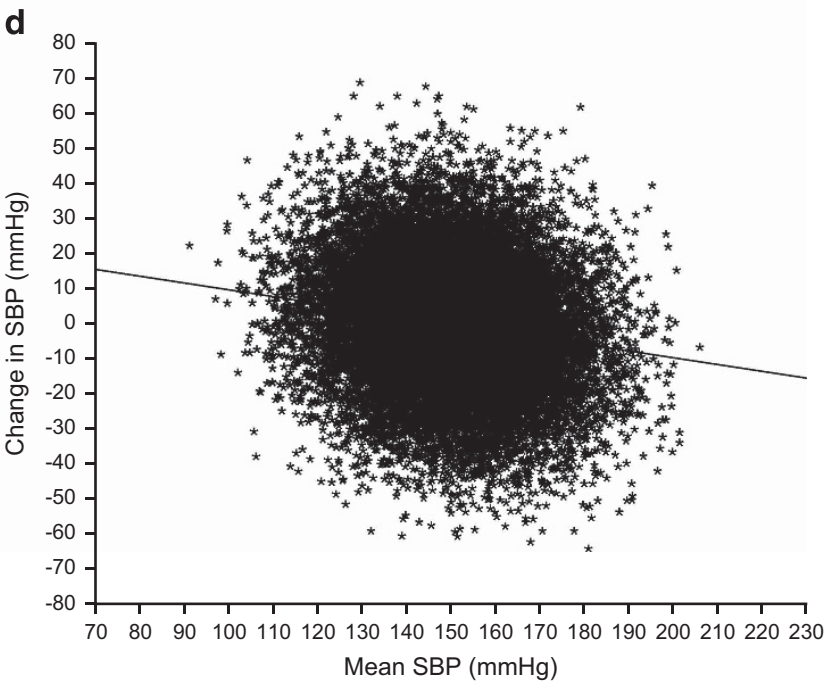

Figure 2 Bland-Altman (BA) plots generated from simulation data with three scenarios for the differences between the s.d. of clinic systolic blood pressure (CSBP) and the s.d. of morning home systolic blood pressure (MHSBP). (a) s.d. of CSBP>s.d. of MHSBP (19.0, 16.4); (b and c) s.d. are equal (19.0, 19.0 and 16.4, 16.4, respectively); (d) s.d. of $\operatorname{CSBP}<$ s.d. of $\operatorname{MHSBP}(16.4,19.0)$. - regression line by univariate regression analysis of the mean of CSBP and MHSBP on the difference between CSBP and MHSBP.

Table 4 Mean CSBP and MHSBP with s.d. in patients with and without a history of cerebro- or cardiovascular disease, concomitant diabetes and CKD

\begin{tabular}{|c|c|c|c|c|c|c|c|c|c|}
\hline & \multicolumn{3}{|c|}{ Cerebro- or cardiovascular disease } & \multicolumn{3}{|c|}{ Concomitant diabetes } & \multicolumn{3}{|c|}{$C K D$} \\
\hline & $\mathrm{n}$ & Mean $(m m ~ H g)$ & s.d. $(m m ~ H g)$ & $\mathrm{n}$ & Mean $(m m ~ H g)$ & s.d. $(m m ~ H g)$ & $\mathrm{n}$ & Mean $(\mathrm{mm} \mathrm{Hg})$ & s.d. $(\mathrm{mm} \mathrm{Hg})$ \\
\hline No & 18828 & 152.1 & 16.4 & 16976 & 152.2 & 16.4 & 16901 & 152.0 & 16.1 \\
\hline Yes & 2241 & 147.0 & 15.9 & 4364 & 149.3 & 16.3 & 4283 & 150.0 & 17.3 \\
\hline Unknown & 271 & 153.2 & 19.2 & & & & 156 & 148.4 & 19.6 \\
\hline \multicolumn{10}{|l|}{$C S B P$} \\
\hline No & 18828 & 154.5 & 18.9 & 16976 & 154.2 & 19.1 & 16901 & 154.2 & 18.7 \\
\hline Yes & 2241 & 146.4 & 17.8 & 4364 & 151.2 & 18.4 & 4283 & 151.6 & 19.9 \\
\hline Unknown & 271 & 153.5 & 23.0 & & & & 156 & 147.4 & 22.9 \\
\hline
\end{tabular}

Abbreviations: CKD, chronic kidney disease; CSBP, clinic systolic blood pressure; MHSBP, morning home systolic blood pressure. 
methods recommended by JSH2009. We did not collect information regarding the home BP device models used by patients. However, the study protocol required use of a home BP measuring device with an upper arm cuff, which are deemed validated and approved by the Ministry of Health, Labour and Welfare of Japan, and meet the standards of United States or European countries. Another limitation is that clinic BP was measured according to the usual methods of each institution under daily clinical practice, and thus not fully standardized. Nevertheless, we consider the influence of those issues to be limited because of the large sample size of the present study. In conclusion, the present analysis clarified the differences between MHSBP and CSBP (i.e. in patients with the mean of CSBP and MHSBP close to $140 \mathrm{~mm} \mathrm{Hg}$, MHSBP was similar to CSBP) in realworld clinical practice. However, the results may vary in different geographic areas, which should be noted in the management of hypertension.

\section{CONFLICT OF INTEREST}

KS, KK, TK, ST and IS have received honoraria from Daiichi Sankyo. KK has received research funding from Daiichi Sankyo. YI is employee of Daiichi Sankyo Co., Ltd. FK is employee of Daiichi Sankyo Development Ltd.

\section{ACKNOWLEDGEMENTS}

We gratefully acknowledge the numerous investigators, fellows, nurses and research coordinators at each of the study sites who have participated in the HONEST study. We also gratefully acknowledge their contribution to the study. The study was supported by funding for data collection and statistical analysis by Daiichi Sankyo (Tokyo, Japan). Statistical analyses were carried out by EPS Corporation (Tokyo, Japan) under the direction of the sponsor and the authors. Medical editorial assistance was provided by Macmillan Medical Communications, Tokyo, Japan, and funded by Daiichi Sankyo Co., Ltd.

1 Sakuma M, Imai Y, Nagai K, Watanabe N, Sakuma H, Minami N, Satoh H, Abe K Reproducibility of home blood pressure measurements over a 1-year period. Am J Hypertens 1997; 10: 798-803.

2 Brueren MM, van Limpt P, Schouten HJ, de Leeuw PW, van Ree JW. Is a series of blood pressure measurements by the general practitioner or the patient a reliable alternative to ambulatory blood pressure measurement? A study in general practice with reference to short-term and long-term between-visit variability. Am J Hypertens 1997; 10 879-885

3 Stergiou GS, Baibas NM, Gantzarou AP, Skeva II, Kalkana CB, Roussias LG, Mountokalakis TD. Reproducibility of home, ambulatory, and clinic blood pressure: implications for the design of trials for the assessment of antihypertensive drug efficacy. Am J Hypertens 2002; 15: 101-104.

4 Sega R, Facchetti R, Bombelli M, Cesana G, Corrao G, Grassi G, Mancia G. Prognostic value of ambulatory and home blood pressures compared with office blood pressure in the general population: follow-up results from the Pressioni Arteriose Monitorate e Loro Associazioni (PAMELA) study. Circulation 2005; 111: 1777-1783.

5 Hara A, Tanaka K, Ohkubo T, Kondo T, Kikuya M, Metoki H, Hashimoto T, Satoh M, Inoue R, Asayama K, Obara T, Hirose T, Izumi S, Satoh H, Imai Y. Ambulatory versus home versus clinic blood pressure: the association with subclinical cerebrovascula diseases: the Ohasama Study. Hypertension 2012; 59: 22-28.

6 Niiranen TJ, Hänninen MR, Johansson J, Reunanen A, Jula AM. Home-measured blood pressure is a stronger predictor of cardiovascular risk than office blood pressure: the Finn-Home study. Hypertension 2010; 55: 1346-1351.

7 SHEP Cooperative Research Group. Prevention of stroke by antihypertensive drug treatment in older persons with isolated systolic hypertension. Final results of the Systolic Hypertension in the Elderly Program (SHEP). JAMA 1991; 265: 3255-3264.

8 Hansson L, Zanchetti A, Carruthers SG, Dahlöf B, Elmfeldt D, Julius S, Ménard J, Rahn KH, Wedel H, Westerling S. HOT Study Group. Effects of intensive blood-pressure lowering and low-dose aspirin in patients with hypertension: principal results of the Hypertension Optimal Treatment (HOT) randomised trial. Lancet 1998; 351 1755-1762.

9 PROGRESS Collaborative Group. Randomised trial of a perindopril-based blood pressure-lowering regimen among 6,105 individuals with previous stroke or transient ischaemic attack. Lancet 2001; 358: 1033-1041.
10 Dahlöf B, Devereux RB, Kjeldsen SE, Julius S, Beevers G, de Faire U, Fyhrquist F, Ibsen H, Kristiansson K, Lederballe-Pedersen O, Lindholm LH, Nieminen MS, Omvik P, Oparil S, Wedel H. LIFE Study Group. Cardiovascular morbidity and mortality in the Losartan Intervention For Endpoint reduction in hypertension study (LIFE) a randomised trial against atenolol. Lancet 2002; 359: 995-1003.

11 ALLHAT Officers and Coordinators for the ALLHAT Collaborative Research Group. Major outcomes in high-risk hypertensive patients randomized to angiotensin-converting enzyme inhibitor or calcium channel blocker vs. diuretic: the Antihypertensive and Lipid-Lowering Treatment to Prevent Heart Attack Trial (ALLHAT). JAMA 2002; 288 2981-2997.

12 Julius S, Kjeldsen SE, Weber M, Brunner HR, Ekman S, Hansson L, Hua T, Laragh J, McInnes GT, Mitchell L, Plat F, Schork A, Smith B, Zanchetti A. VALUE trial group. Outcomes in hypertensive patients at high cardiovascular risk treated with regimens based on valsartan or amlodipine: the VALUE randomised trial. Lancet 2004; 363 . 2022-2031.

13 Kabutoya T, Ishikawa J, Hoshide S, Eguchi K, Ishikawa S, Shimada K, Kario K. Determinants of negative white-coat effect in treated hypertensive patients: the Jichi Morning Hypertension Research (J-MORE) study. Am J Hypertens 2009; 22 $35-40$

14 Verberk WJ, Kroon AA, Kessels AG, de Leeuw PW. Home blood pressure measurement: a systematic review. J Am Coll Cardiol 2005; 46: 743-751.

15 Guidelines Subcommittee. 1999 World Health Organization-International Society of Hypertension Guidelines for the Management of Hypertension. J Hypertens 1999; 17: 151-183.

16 Mancia G, Fagard R, Narkiewicz K, Redón J, Zanchetti A, Böhm M, Christiaens T, Cifkova R, De Backer G, Dominiczak A, Galderisi M, Grobbee DE, Jaarsma T, Kirchho P, Kjeldsen SE, Laurent S, Manolis AJ, Nilsson PM, Ruilope LM, Schmieder RE, Sirnes PA, Sleight P, Viigimaa M, Waeber B, Zannad F. Task Force Members. 2013 ESH/ESC Guidelines for the Management of Arterial Hypertension: the Task Force for the Management of Arterial Hypertension of the European Society of Hypertension (ESH) and of the European Society of Cardiology (ESC). J Hypertens 2013; 31: 1281-1357.

17 Ogihara T, Kikuchi K, Matsuoka H, Fujita T, Higaki J, Horiuchi M, Imai Y, Imaizumi T, Ito S, Iwao H, Kario K, Kawano Y, Kim-Mitsuyama S, Kimura G, Matsubara H, Matsuura H, Naruse M, Saito I, Shimada K, Shimamoto K, Suzuki H, Takishita S, Tanahashi N, Tsuchihashi T, Uchiyama M, Ueda S, Ueshima H, Umemura S, Ishimitsu T, Rakugi H. Japanese Society of Hypertension Committee. The Japanese Society of Hypertension Guidelines for the Management of Hypertension (JSH 2009). Hypertens Res 2009; 32: 3-107

18 Saito I, Kario K, Kushiro T, Teramukai S, Zenimura N, Hiramatsu K, Kobayashi F, Shimada K. Rationale, study design, baseline characteristics and blood pressure at 16 weeks in the HONEST Study. Hypertens Res 2013; 36: 177-182.

19 Kario K, Saito I, Kushiro T, Teramukai S, Ishikawa Y, Mori Y, Kobayashi F, Shimada K. Home blood pressure and cardiovascular outcomes in patients during antihypertensive therapy primary results of HONEST, a large-scale prospective, real-world observational study. Hypertension 2014; 64: 989-996.

20 Association for the Advancement of Medical Instrumentation. American Nationa Standard. Electronic or Automated Sphygmomanometers. NSI/AAMI SP 10-1992. AAMI, Arlington, VA, 1993, p 40.

21 European Committee for Standardization. Non-invasive Sphygmomanometers. Part 3 Supplementary Requirements for Electromechanical Blood Pressure Measuring Systems. British Standard BS EN 1060-3: 1997. European Standard EN 1060-3 1997. ECS, Brussels, 1997.

22 Bland JM, Altman DG. Statistical methods for assessing agreement between two methods of clinical measurement. Lancet 1986; 1: 307-310.

23 Bland JM, Altman DG. Measuring agreement in method comparison studies. Stat Methods Med Res 1999; 8: 135-160.

24 Niiranen TJ, Jula AM, Kantola IM, Reunanen A. Comparison of agreement between clinic and home-measured blood pressure in the Finnish population: the Finn-HOME Study. J Hypertens 2006; 24: 1549-1555.

25 Horikawa T, Obara T, Ohkubo T, Asayama K, Metoki H, Inoue R, Kikuya M, Hashimoto J, Totsune K, Imai Y. J-HOME Study Group. Difference between home and office blood pressures among treated hypertensive patients from the Japan Home versus Office Blood Pressure Measurement Evaluation (J-HOME) study. Hypertens Res 2008; 31: 1115-1123.

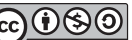

This work is licensed under a Creative Commons Attribution-NonCommercial-ShareAlike $\quad 4.0$ International License. The images or other third party material in this article are included in the article's Creative Commons license, unless indicated otherwise in the credit line; if the material is not included under the Creative Commons license, users will need to obtain permission from the license holder to reproduce the material. To view a copy of this license, visit http://creativecommons.org/licenses/ by-nc-sa/4.0/

Supplementary Information accompanies the paper on Hypertension Research website (http://www.nature.com/hr) 